\title{
Hypoxic-mediated oxidative stress condition and hydroxyapatite-inducing osteogenic differentiation of human mesenchymal stem cells: a mathematical modelling study
}

\begin{abstract}
Avascular necrosis (AVN) of the bones remains a major clinical challenge. Fractures in the talus, the scaphoid, and the neck of the femur are especially challenging to heal due to the low blood vessel network and the lack of collateral blood supply. These fractures are associated with high rates of nonunion and increased infections that require repeated operations. Conventional treatments by autografting or allografting bone replacement and synthetic bone implants have limitations, including the invasiveness of operative procedures, tissue supply insufficiency, and the risk of host rejection. The advancement in tissue engineering has revealed the potential of stem cells as restorative agents for bone injuries. The administration of mesenchymal stem cells (MSCs) into the talus, the scaphoid, and the neck of the femur could produce enhanced osteogenesis via the manipulation of MSC culture conditions. In this study, we used hydroxyapatite as the nanomaterial, and hypoxic milieu to enhance MSC differentiation capacity into the osteogenic lineage, allowing for more rapid and efficient bone cell replacement treatment. Our results demonstrate $1 \%$ oxygen and $12.5 \mu \mathrm{g} / \mathrm{mL}$ of hydroxyapatite (HAP) as the optimal conditions to incorporate the osteogenic medium for the osteogenic induction of MSCs. We also established a proof of concept that the addition of HAP and hypoxic conditions could augment the osteoinductive capacity of MSCs. We also developed an accurate mathematical model to support future bone cell replacement therapy.
\end{abstract}

Keyword: Hydroxyapatite; Hypoxia; Mathematic modeling; Osteogenesis; Stem cells 